\title{
ESTUDO COMPARATIVO DAS PROPRIEDADES QUÍMICAS DO RESÍDUO BORRA OLEOSA ASFÁLTICA E DO LIGANTE ASFÁLTICO DE PETRÓLEO
}

\author{
P. R. N. Fernandes ${ }^{1}$, A. E. V. de Alencar, J. B. Soares, R. M. Cavalcante, R. F. do Nascimento, S. A. Soares, M. D. T. \\ Casagrande \\ Universidade Federal do Ceará - UFC \\ E-mail: paulo.fernandes@ifrn.edu.br ${ }^{1}$
}

Artigo submetido em março/2013 e aceito em agosto/2013

\section{RESUMO}

Neste trabalho, as principais características químicas do resíduo borra oleosa asfáltica foram estudadas e comparadas com as propriedades do ligante asfáltico correspondente. A espectroscopia de ressonância magnética nuclear e os espectros de infravermelho com transformada de Fourier indicaram a presença de grupos alifáticos, aromáticos, carbonilas e sulfóxidos. A curva termogravimétrica mostrou que o resíduo inicia a decomposição térmica a uma temperatura mais elevada, provavelmente devido ao baixo teor de fração volátil maltênica. Hidrocarbonetos policíclicos aromáticos (HPAs) foram extraídos a partir da fração maltênica utilizando métodos cromatográficos tais como: a dispersão de matriz em fase sólida, por cromatografia de adsorção em coluna e cromatografia em fase gasosa. Os resultados indicam que os materiais apresentam HPAs tóxicos, bem como, mutagênicos e carcinogênicos.

PALAVRAS-CHAVE: Resíduo Borra Oleosa Asfáltica, Cimento Asfáltico, Análise Química.

\section{A COMPARATIVE STUDY OF THE CHEMICAL PROPERTIES OF ASPHALT BINDER OIL RESIDUE AND ASPHALT BINDER}

\section{ABSTRACT}

In this work, the main chemical characteristics of asphalt binder oil residue were studied and compared to the properties of the corresponding asphalt binder. The Nuclear magnetic resonance and Fourier transform infrared spectroscopy spectra indicated the presence of aliphatic groups, aromatics, carbonyls and sulfoxides. The thermogravimetric curve showed that the residue begins its thermal decomposition at a higher temperature, probably due to lower content of maltenic fraction. Hydrocarbons polycyclic aromatics (HPAs) were extracted from the maltene fraction using chromatographic methods such as: matrix dispersion in solid phase, chromatographic adsorption in column and gas chromatographic. The results indicate that the materials present toxic HPAs, as well as, mutagenic and carcinogenic.

KEYWORDS: Asphalt Binder Oil Residue, Asphalt Binder, Chemical Analysis. 


\section{A COMPARATIVE STUDY OF THE CHEMICAL PROPERTIES OF ASPHALT BINDER OIL RESIDUE AND ASPHALT BINDER}

\section{INTRODUÇÃO}

Em todas as operações, a indústria petrolífera gera resíduos oleosos de diversos tipos, desde a perfuração, passando pelas etapas de produção, armazenamento, transporte e refino até a distribuição dos derivados. Estes derivados apresentam-se potencialmente poluidores, pelo volume, concentração e toxicidade dos resíduos gerados [1,2]. Diante deste fato, faz-se necessário desenvolver novas metodologias que permitam mitigar os impactos ambientais das atividades da indústria petrolífera.

As refinarias respondem pela maior parte dos resíduos gerados na indústria do petróleo, dos quais se destacam os produtos acumulados no fundo dos tanques de óleo cru, lodos oleosos, lodos das torres de resfriamento, catalisadores gastos, resíduos das torres de troca de calor, finos de coque e águas residuárias [3]. Dentre os resíduos gerados no fluxo e processos produtivos do setor petrolífero, o mais abundante é a borra oleosa, formada durante as etapas de produção, transporte e refino [4]. As borras se constituem de emulsões, basicamente compostas por óleo, água e sólidos grosseiros [5]. Devido à presença de resíduo químico tóxico, a borra é classificada, segundo a NBR 10004 [6], como um resíduo classe I-perigoso, portanto, o mesmo não pode ser lançado diretamente no meio ambiente ou comercializado.

A grande variedade de resíduos sólidos e semi-sólidos gerados nas unidades de uma refinaria tem sido cientificamente pouco investigada no Brasil e no exterior. Buscam-se novas aplicações para a borra asfáltica na área de misturas de revestimento, visando à pavimentação de baixo custo e baixo volume de tráfego. A experiência acumulada com este tipo de material até o presente momento se restringe a técnicas de tratamento ambiental do resíduo, podendo este ser usado, após tratado, como material de construção em sistemas de impermeabilização de aterros sanitários, por exemplo.

Entre os constituintes mais perigosos podemos destacar os hidrocarbonetos policíclicos aromáticos (HPAs), alguns dos quais considerados cancerígenos e/ou mutagênicos. Os HPAs formam uma classe de compostos orgânicos, constituídos de 2 a 7 anéis benzênicos fundidos, que podem ser de origem natural, produzidos nas queimadas ou por microorganismos em seu metabolismo, ou de origem antropogênica. A indústria petrolífera e a queima de combustíveis fósseis são as principais fontes de produção dos HPAs [7]. Dados indicam que a emissão gerada durante a aplicação do cimento asfáltico de petróleo (CAP), especialmente quando usado na produção de misturas asfálticas a quente, pode conter um grande número de compostos, entre os quais os HPAs, potencialmente prejudiciais à saúde dos profissionais envolvidos nestes procedimentos $[8,9]$.

No presente estudo, o resíduo (borra oleosa asfáltica) foi cedido pela refinaria Lubrificantes do Nordeste (Lubnor)/Petrobras, localizada em Fortaleza-Ceará, na região Nordeste do Brasil, onde 56,8 toneladas de borra de tanque foram geradas no ano de 2008 . Em 2010 foram geradas 400,1 toneladas de borra. Ou seja, a geração deste tipo de resíduo é variável no tempo e depende também da capacidade de produção e geração de borra nas refinarias, estando sujeita à frequência na manutenção dos tanques de armazenamento, a problemas de estocagem do resíduo e a problemas operacionais. No ano de 2004, a refinaria em questão processava inicialmente 250.076,4 toneladas de petróleo e em 2010 possuiu uma produção anual potencial 
de 461.040 toneladas, após passar por várias ampliações e instalação de novas unidades. A busca por tratamentos eficazes que possam ser implementados a um custo acessível é um problema de difícil solução dentro do programa de gerenciamento de resíduos da indústria petrolífera. 0 método empregado atualmente pela Lubnor para o tratamento da borra utilizada nesta pesquisa é o co-processamento. Trata-se do processo de combustão do resíduo oleoso, em fornos de indústrias cimenteiras, utilizado para eliminar o conteúdo orgânico do mesmo, incorporando as cinzas ao cimento produzido. O processo se dá em uma indústria localizada em Sobral, a $233 \mathrm{~km}$ de Fortaleza, resultando em um custo de aproximadamente $\mathrm{R} \$ 490,00$ para a refinaria, por tonelada de resíduo gerado, sendo já incluso custos de transporte.

Os CAPs são misturas complexas com uma considerável variedade de compostos orgânicos. As duas principais frações são formadas pelos maltenos, constituídos pelos compostos saturados, aromáticos e resinas, e os asfaltenos, que constituem a fração mais pesada e polar do CAP asfáltico. Estes componentes formam uma estrutura coloidal: micelas de asfaltenos estabilizados pelas resinas adsorvidas na superfície, dispersos numa fase maltênica.

Este trabalho visa avaliar a borra oleosa asfáltica e o CAP convencional (CAP50/70). Sob o ponto de vista das características químicas foram utilizados: espectroscopia no infravermelho (FTIR), ressonância magnética nuclear (RMN), termogravimetria (TG) e cromatografia de permeação em gel (GPC). Com a finalidade de avaliar os HPAs presentes na fração maltênica do CAP foram utilizados métodos cromatográficos, como: dispersão de matriz em fase sólida (DMFS), cromatografia de adsorção em coluna e cromatografia gasosa (GC) para a determinação qualitativa e quantitativa dos HPAs.

\section{MATERIAS E METODOS}

\section{Materiais}

Como CAP utilizou-se o CAP 50/70 fornecido pela Lubnor/Petrobras. Este é proveniente do petróleo nacional Fazenda Alegre e atendeu a todas as especificações da Agência Nacional do Petróleo, Gás Natural e Biocombustíveis.

O resíduo utilizado, também conhecido como borra oleosa asfáltica pura, é proveniente do acúmulo de material retirado do fundo dos tanques de armazenamento de CAP, localizados na Lubnor/Petrobras e também foi cedido pela empresa.

Os solventes orgânicos (tolueno, hexano, acetato de etila, piridina) utilizados na pesquisa são da marca Synth e foram bidestilados de forma a se obter um solvente mais puro. Os padrões analíticos, da marca Supelco, empregados foram os seguintes: antraceno, benzo(a)antraceno, naftaleno, m-naftaleno, fluoreno, fenantreno, criseno, benzo(b)fluoranteno, antraceno, benzo(k)fluoranteno, benzo(a)pireno, dibenzo(a,h)antraceno, perileno, pireno, indeno(c, d)pireno, dibenzo(a,h)antraceno, benzo(g, h, i)perileno. O padrão interno, 2-flúor-bifenil, foi obtido da Sigma Aldrich.

\section{Metodologia}

\section{Extração dos Asfaltenos e Maltenos}


A extração dos asfaltenos do CAP e da borra asfáltica foi realizada através de uma adaptação da norma IP-143 [10], que descreve a metodologia para quantificação destas frações pesadas em petróleo. O método para a extração consistiu em misturar 5,0g de amostra (CAP e borra oleosa) com o solvente $n$-hexano na razão 1:40. As soluções foram agitadas por 1 hora em um agitador de bancada QUIMIS. Em seguida, os materiais foram retirados do agitador e filtrados em filtro Whatman $\mathrm{N}^{\circ} 1$. Os sólidos retidos no filtro foram submetidos a lavagens sucessivas com n-hexano. Após algumas lavagens, o n-hexano foi substituído por tolueno para a extração dos asfaltenos. Em seguida, o tolueno foi evaporado a uma temperatura de $70 \circ \mathrm{C}$. Os maltenos foram recuperados a partir da evaporação do n-hexano, da solução residual, a uma temperatura de $40^{\circ} \mathrm{C}[11]$.

\section{Extração dos HPAs}

As extrações das fases maltênicas do CAP Fazenda Alegre e da borra oleosa foram realizadas por agitação em bancada (batelada). Foi utilizado 0,3g de amostra (CAP e borra oleosa), ambos dopados com o padrão surrogate e $12,0 \mathrm{~mL}$ de $\mathrm{n}$-hexano. A mistura foi agitada por 2 horas em um agitador de bancada QUIMIS. Após agitação, a mistura foi filtrada a vácuo e a solução recolhida (fase maltênica). Em seguida, foi pré-concentrada para aproximadamente $2,0 \mathrm{~mL}[12,13]$.

As soluções maltênicas foram submetidas ao método DMFS (Dispersão da Matriz em Fase Sólida) a fim de avaliar a presença de HPAs. Tal método consistiu em dispersar aproximadamente $2,0 \mathrm{~mL}$ da solução maltênica em $15,0 \mathrm{~g}$ de sílica 60 mesh. Depois da dispersão a mistura foi homogeneizada e percolada em uma coluna de $50,0 \mathrm{~cm} \times 1,0 \mathrm{~cm}$, com 5,0g de alumina. Em seguida, procedeu-se a lavagem da amostra com $50,0 \mathrm{~mL}$ de $\mathrm{n}$-hexano, retirando a fase dos compostos alifáticos presentes nas amostras $[12,13]$.

Após a lavagem realizou-se a eluição da fração com compostos aromáticos a fim de separá-los tanto do CAP quanto da borra, separadamente. A eluição consistiu em passar pela coluna três frações de solventes: $F 1$ (36,0mL de $n$-hexano $+4,0 m L$ de acetato de etila), F2 $(16,0 \mathrm{~mL}$ de $\mathrm{n}$-hexano $+4,0 \mathrm{~mL}$ de acetato de etila) e $\mathrm{F} 3(14,0 \mathrm{~mL}$ de $\mathrm{n}$-hexano $+6,0 \mathrm{~mL}$ de acetato de etila). O aumento na polaridade das fases utilizadas para a eluição dos HPAs deve-se a variação de polaridade dos HPAs de interesse $[12,13]$. Os valores utilizados na mistura foram estabelecidos durante os ensaios, buscando encontrar uma faixa de polaridade ótima para a extração [14]. Após esta etapa, a fração dos aromáticos foi recolhida, rotoevaporada e préconcentrada para $0,5 \mathrm{~mL}$, sendo então adicionados $10,0 \mu \mathrm{L}$ de padrão interno.

\section{Análise Cromatográfica para Identificação dos HPAs}

Foi utilizado um cromatógrafo gasoso, modelo CG17A-Shimadzu, interfaciado com um detector de ionização por chama (DIC), acoplado com uma coluna DB-5 J\&W Scientific (30m $\times$ $0,25 \mathrm{~mm}$ d.i. $\times 0.25 \mu \mathrm{m}$ de filme). As amostram foram injetadas em triplicata no modo split (1:20), utilizando hidrogênio como gás de arraste, a um fluxo de $1,0 \mathrm{~mL} / \mathrm{min}$. As temperaturas do injetor e detector foram de $280^{\circ} \mathrm{C}$ e $300^{\circ} \mathrm{C}$, respectivamente.

Segundo Lanças [15], não há consenso sobre quais parâmetros devem ser incluídos em um processo de validação de um método analítico. Para a validação [16] principalmente da 
eficiência de separação e quantificação dos HPAs na coluna DB-5 J\&W Scientific, foram empregados os parâmetros de identificação e confirmação dos constituintes, além da precisão, fidelidade, linearidade, seletividade e limite de deteç̧ão.

A linearidade é a resposta obtida em função da concentração do analito, a qual deve ser estudada em um intervalo de concentração apropriado. Geralmente é determinada por intermédio de gráficos de calibração (curva de calibração) os quais são produzidos com 5 a 6 pontos. Foram construídas curvas de calibração com os pontos 0,$5 ; 2,5 ; 5 ; 10 ; 20 ; 50$ e 100 $\mathrm{gg} / \mathrm{mL}$, as quais foram utilizadas na quantificação dos HPAs. O coeficiente de correlação $(R)$ variou de 0,9899 a 0,9989. O limite mínimo de deteç̧ão variou de 0,2 a $0,5 \mu \mathrm{g} / \mathrm{mL}$ [17]. A confirmação dos HPAs estudados foi através de um cromatógrafo a gás CG 17A acoplado a um detector de espectrometria de massa (marca Shimadzu GCMSQP5000), utilizando também uma biblioteca de dados NIST.

\section{Cálculo da Massa Molar}

A análise da massa molar da borra e do CAP foi realizada através da cromatografia de permeação em gel (GPC). Utilizou-se um sistema cromatográfico tipo HPLC, constituído de uma bomba modelo LC-10AD funcionando isocraticamente com um detector de índice de refração modelo RID-6A, ambos da Shimadzu. A amostra e os padrões foram injetados em alíquotas de $50 \mu \mathrm{L}$. Foram empregadas uma pré-coluna e duas colunas em série da Phenomenex, do tipo Phenogel LINEAR/MIXED 5 e $5 \mathrm{U}$ com $7,80 \times 50 \mathrm{~mm}$ e $7,8 \times 300 \mathrm{~mm}$, respectivamente, ambas empacotadas com o copolímero estireno-divinilbenzeno (SDVB). A fase móvel empregada foi o tolueno com o fluxo de $1 \mathrm{~mL} / \mathrm{min}$. A curva de calibração para a determinação da massa molar depico (Mpk) foi construída utilizando padrões de poliestireno (Shodex-Showadenko), com as seguintes concentrações: c = 0,10\% ( $\overline{M w}=1,13 \times 103 \mathrm{~g} / \mathrm{mol} ; \bar{M} w=2,96 \times 103 \mathrm{~g} / \mathrm{mol}), \mathrm{c}=0,08 \%$ ( $\bar{M} w=2,85 \times 104 \mathrm{~g} / \mathrm{mol} ; \bar{M} w=6,60 \times 104 \mathrm{~g} / \mathrm{mol} ; \bar{M} w=1,72 \times 105 \mathrm{~g} / \mathrm{mol} ; \bar{M} w=5,65 \times 105 \mathrm{~g} / \mathrm{mol})$, c $=0,05 \%(\bar{M} w=1,01 \times 106 \mathrm{~g} / \mathrm{mol} ; \bar{M} w=2,16 \times 106 \mathrm{~g} / \mathrm{mol})$.

\section{Caracterização Estrutural por Infravermelho (FTIR) e Ressonância Magnética Nuclear (RMN)}

Os espectros FTIR do CAP 50/70 e da borra asfáltica foram obtidos no espectrômetro Shimadzu FTIR-8300 na forma de discos de $\mathrm{KBr}$ e analisados na região de 600 à $4000 \mathrm{~cm}^{-1}$. Os espectros de RMN $1 \mathrm{H}$ dos mesmos materiais foram obtidos em solução de clorofórmio deuterado através do espectrômetro Brucker DRX-500, operando a $500 \mathrm{MHz}$ na frequência do $1 \mathrm{H}$.

\section{Análise Termogravimétrica (TGA)}

A TGA foi utilizada para o estudo da estabilidade térmica do CAP e da borra asfáltica. Todos os experimentos foram realizados em um sistema Shimadzu TGA-50 utilizando-se atmosfera oxidativa com fluxo de $50 \mathrm{~mL} / \mathrm{min}$. Aproximadamente $10 \mathrm{mg}$ de amostra foram aquecidos de 25 a $800^{\circ} \mathrm{C}$ a uma razão de aquecimento de $10^{\circ} \mathrm{C} / \mathrm{min}$. 


\section{RESULTADOS E DISCUSSÕES}

\section{Fração Maltênica}

\section{Extração e Identificação dos HPAs}

Na Tabela 1 encontram-se os dados obtidos na identificação dos HPAs presentes no CAP e na borra oleosa, extraídos da fração maltênica e o tempo de retenção de 16 HPAs prioritários sugeridos pela EPA (Environmental ProtectionAgency).

Tabela 1- Valores de concentração dos HPAs presentes no CAP e na borra com o uso do agitador de bancada.

\begin{tabular}{ccccccc}
\hline & \multicolumn{2}{c}{$\mathbf{p p m}$} & \multicolumn{2}{c}{$\begin{array}{c}\text { Desvio } \\
\text { Padrão }\end{array}$} & Erro relativo (\%) \\
(mg.kg & & \multicolumn{2}{c}{ ) } & & \\
\cline { 2 - 6 } HPAs & CAP & Borra & CAP & Borra & CAP & Borra \\
\hline Antraceno & 5,90 & 8,28 & 1,34 & 1,38 & 22,65 & 19,67 \\
Benzo(a)Antraceno & 6,49 & 7,39 & 1,34 & 2,20 & 20,64 & 17,34 \\
Benzo(a)Pireno & 14,75 & 17,39 & 2,54 & 2,36 & 17,20 & 16,98 \\
Benzo(b)Fluoranteno & 15,78 & 6,14 & 2,64 & 2,25 & 16,69 & 16,15 \\
Benzo(k)Fluoranteno & 9,58 & 9,46 & 1,23 & 1,14 & 12,80 & 12,11 \\
Benzo(g,h,i)Perileno & 16,93 & 20,67 & 19,26 & 9,02 & 17,95 & 18,52 \\
Criseno & 6,88 & 6,38 & 1,37 & 1,56 & 19,97 & 19,22 \\
Dibenzo (a,h)Antraceno & 12,56 & 18,43 & 10,96 & 10,49 & 16,10 & 15,36 \\
Fenantreno & 5,55 & 6,23 & 1,33 & 1,35 & 23,89 & 21,56 \\
Fluoranteno & 6,77 & 5,93 & 0,93 & 0,89 & 13,69 & 10,36 \\
Fluoreno & 6,76 & 3,33 & 1,56 & 1,78 & 23,07 & 22,48 \\
Indeno (c,d)Pireno & 14,94 & 15,86 & 15,60 & 8,37 & 17,80 & 16,39 \\
Naftaleno & 3,24 & 4,30 & 0,11 & 0,18 & 3,24 & 3,78 \\
m-Naftaleno & 4,93 & 3,69 & 0,20 & 0,35 & 4,93 & 4,26 \\
Pireno & 6,01 & 11,69 & 0,93 & 1,72 & 15,43 & 15,56 \\
Perileno & 11,61 & 13,27 & 3,41 & 3,02 & 27,41 & 25,36 \\
\hline
\end{tabular}

Os dados referentes ao CAP encontram-se reportados na literatura $[12,13,17]$. Com base na Tabela 1, observa-se que a técnica de agitação em bancada é eficiente na extração dos HPAs, uma vez que foram observados 14 HPAs prioritários, além do perileno e do m-naftaleno. Tal classificação baseia-se nas características tóxicas, mutagênicas e carginogênicas: HPAs de até 3 anéis são considerados tóxicos, enquanto HPAs com 4 ou mais anéis são considerados mutagênicos ou carcinogênicos. Nas Tabelas 2 e 3 encontram-se mais detalhes do potencial carcinogênico em humanos e animais de alguns HPAs [18-20].

Tabela 2- Classificação de HPAs quanto a carcinogenicidade pela IARC e USEPA [18,19].

\begin{tabular}{ccc}
\hline Classificação da IARC & Classificação pela USEPA & Hidrocarboneto \\
\hline 3 & $\mathrm{P}$ & Antraceno \\
2A & $\mathrm{P}$ & Benzo(a)Antraceno \\
2A & $\mathrm{P}$ & Benzo(a)Pireno \\
3 & - & Benzo(e)Pireno \\
2B & $\mathrm{P}$ & Benzo(b)Fluoranteno \\
2B & - & Benzo(j)Fluoranteno \\
\hline
\end{tabular}




\begin{tabular}{ccc}
\hline 2B & P & Benzo(k)Fluoranteno \\
3 & $\mathrm{P}$ & Benzo(g,h,i)Perileno \\
3 & $\mathrm{P}$ & Criseno \\
$2 \mathrm{~A}$ & $\mathrm{P}$ & Dibenzo(a,h)Antraceno \\
$2 \mathrm{~B}$ & - & Dibenzo(a,e)Pireno \\
$2 \mathrm{~B}$ & - & Dibenzo(a,h)Pireno \\
$2 \mathrm{~B}$ & - & Dibenzo(a,i)Pireno \\
$2 \mathrm{~B}$ & - & Dibenzo(a,l)Pireno \\
3 & $\mathrm{P}$ & Fenantreno \\
3 & $\mathrm{P}$ & Fluoranteno \\
3 & $\mathrm{P}$ & Fluoreno \\
2B & $\mathrm{P}$ & Indeno $(1,2,3-\mathrm{c}, \mathrm{d})$ \\
& & Pireno \\
2B & $\mathrm{P}$ & Naftaleno \\
3 & $\mathrm{P}$ & Pireno \\
\hline
\end{tabular}

Classificação pela IARC: $2 \mathrm{~A}=$ provavelmente cancerígeno ao homem; $2 \mathrm{~B}=$ possivelmente cancerígeno ao homem; $3=$ não classificado como cancerígeno ao homem; $4=$ provavelmente não é cancerígeno ao homem. Classificação pela USEPA: $P=$ prioritário.

Tabela 3- Limite recomendado dos HPAs $[19,20]$.

\begin{tabular}{ccc}
\hline Limite Recomendado & Portaria 518 & EPA \\
\hline Antraceno $\left(\mu \mathrm{g} . \mathrm{L}^{-1}\right)$ & - & 1800 \\
Fluoranteno $\left(\mu \mathrm{g} \cdot \mathrm{L}^{-1}\right)$ & - & 1500 \\
Benzo(a) antraceno $\left(\mu \mathrm{g} . \mathrm{L}^{-1}\right)$ & - & 0,092 \\
Criseno $\left(\mu \mathrm{g} . \mathrm{L}^{-1}\right)$ & - & 0,56 \\
Benzo(b) fluoranteno $\left(\mu \mathrm{g} . \mathrm{L}^{-1}\right)$ & - & 0,092 \\
Benzo(k) fluoranteno $\left(\mu \mathrm{g} \cdot \mathrm{L}^{-1}\right)$ & - & 0,56 \\
Benzo(a)pireno $\left(\mu \mathrm{g} \cdot \mathrm{L}^{-1}\right)$ & 0,7 & 0,0092 \\
\hline
\end{tabular}

Além dos HPAs prioritários, as amostras apresentaram picos diferentes dos padrões analíticos utilizados nos experimentos. Tais picos podem estar associados aHPAs com organização dos anéis diferentes dos padrões utilizados, ou HPAs com cadeias alifáticas substituintes $[12,13]$.

A borra oleosa apresentou em sua composição:

a) um teor maior de alguns HPAs (naftaleno, fenantreno, antraceno, pireno, benzo(a)antraceno, benzo(a)pireno, perileno, indeno(c,d)pireno, dibenzo(a,h)antraceno e benzo(g,h,i)perileno quando em comparação ao CAP (Tabela 1), principalmente os HPAs com 4 ou mais anéis aromáticos;

b) uma redução no teor de m-naftaleno, fluoreno, fluoranteno, criseno, benzo(b) fluoranteno, benzo(k)fluoranteno quando comparado com o CAP.

Isso pode estar associado ao fato de a borra ser um resíduo pré-concentrado, que apresenta uma grande quantidade de aromáticos e, neste caso um teor muito alto de HPAs, usualmente encontrada neste tipo de resíduo. 
Os valores de concentração dos HPAs encontrados tanto no CAP, quanto na borra oleosa, apresentam-se em valores superiores aos recomendados na Portaria № 518 do Ministério da Saúde [21] e pela EPA [19]. Conforme indicado no material suplementar, tanto o CAP quanto a borra podem representar um risco potencial a saúde humana, se manuseados sem os equipamentos de segurança individual (EPI), principalmente nos processos de usinagem e compactação, onde a liberação de compostos orgânicos voláteis é maior, pois os materiais são expostos a temperaturas elevadas.

\section{Análise de Massa Molar}

A medida da massa molar do CAP e da borra foi realizada através da cromatografia de permeação em gel (GPC). Os cromatogramas obtidos são mostrados na Figura 1 e os valores de volume de eluição (Ve) e massa molar de pico (Mpk) encontram-se apresentados na Tabela 4. Com base na Equação 1, foi possível calcular a massa molar do CAP e da borra oleosa.

$$
\log M p K=-0,38(V e)+10,68
$$

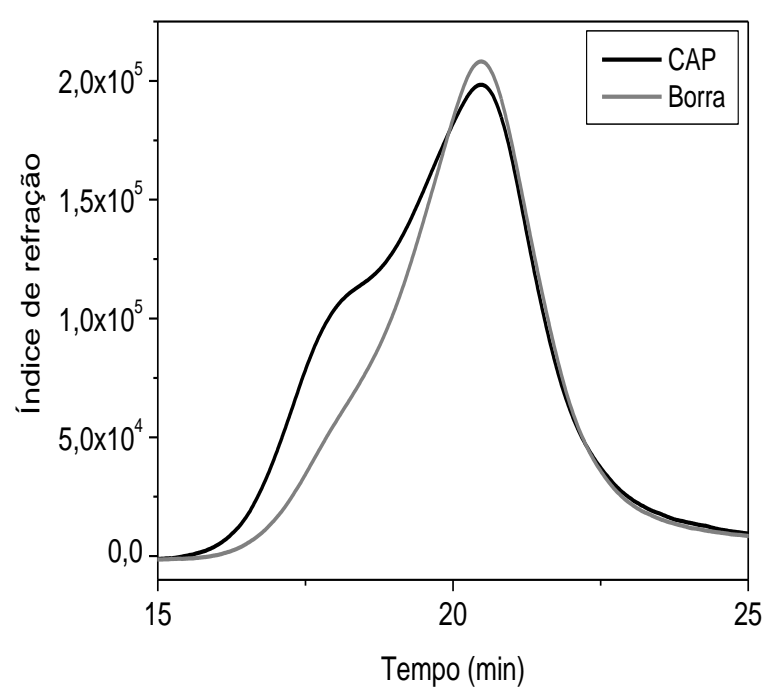

Figura 1-Cromatograma do CAP e da borra asfáltica.

A partir dos resultados mostrados nos cromatogramas da Figura 1, observou-se que tanto o CAP quanto para a borra apresentaram teores de compostos de alta e de baixa massa molar. Os asfaltenos, como esperado, representaram a fração de maior massa molar nos dois materiais.

Tabela 4- Valores de volume de eluição e Mpk do CAP e da borra.

\begin{tabular}{ccc}
\hline Amostra & Volume de eluição $(\mathrm{mL})$ & Mpk $(\mathrm{g} / \mathrm{mol})$ \\
\hline CAP & 17,9 & $7,1 \times 10^{3}$ \\
& 20,4 & $7,9 \times 10^{2}$ \\
Borra & 17,9 & $7,4 \times 10^{3}$ \\
& 20,4 & $8,1 \times 10^{2}$ \\
\hline
\end{tabular}


Observou-se que os tempos de eluição para a borra foram menores do que para o CAP e, consequentemente, as moléculas presentes na borra apresentaram maiores raios hidrodinâmicos (moléculas maiores) e uma maior velocidade de deslocamento dentro da coluna cromatográfica. Este fato pode estar associado ao processo de produção do CAP, já que a borra é um resíduo gerado dos processos de operacionalização do sistema produtivo do CAP, e como consequência, uma mudança na concentração e composição química dos constituintes do asfalto é esperada, tornando a estrutura mais complexa. A complexidade das amostras analisadas dificultou a eficiência de separação, consequentemente, os cromatogramas não apresentaram boa seletividade e pequeno alargamento das bandas.

\section{Ressonância Magnética Nuclear}

Os espectros de RMN $1 \mathrm{H}$ para o CAP e a borra são mostrados na Figura 2.

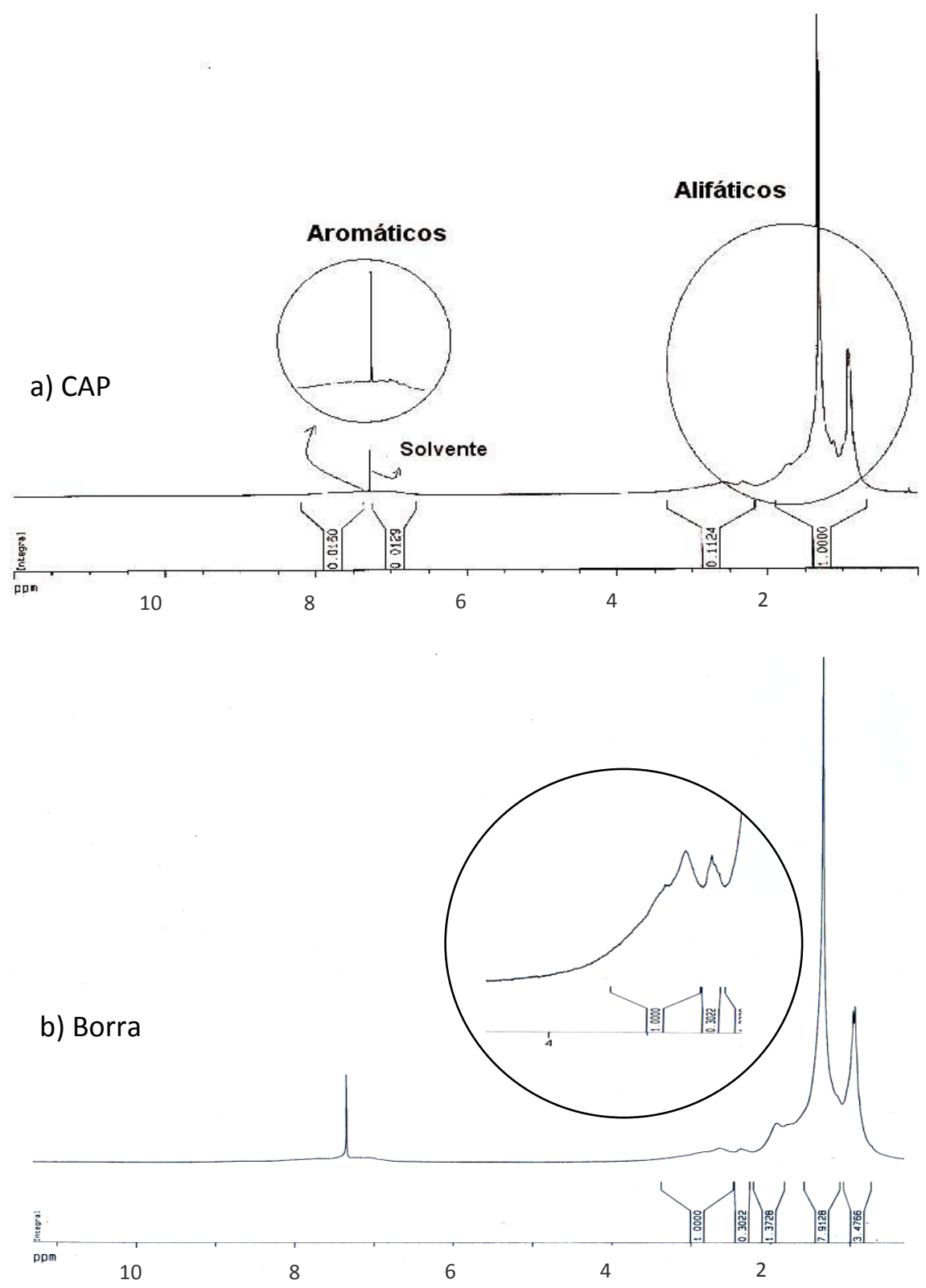

Figura 2- Espectro RMN 13C GATED inverso do (a) CAP e da (b) Borra. 
Nos espectros RMN $1 \mathrm{H}$ a região de 1 a $5 \mathrm{ppm}$ foi atribuída à região de prótons de natureza alifática e a região de 6 a 8 ppm foi atribuída à região de natureza aromática [22], sendo a região de aromáticos mais difícil de analisar devido a grande superposição dos picos. Este mesmo comportamento é observado em outros estudos [23]. A análise dos espectros do CAP e da borra não apresentou picos bem definidos. A razão é devido à natureza das amostras apresentarem uma mistura complexa de compostos orgânicos. Como consequência, não são observadas diferenças estruturais quando da comparação das amostras [24, 25].

Para verificar diferenças na estrutura do CAP e da borra utilizou-se a técnica de GATED inverso $13 \mathrm{C}$ para o cálculo de parâmetros (\% carbono aromáticos e \% carbono alifáticos). 0 resultado dos valores de percentagem de carbono aromático e alifático é mostrado na Tabela 5.

Tabela 5- Valores de carbono aromático e alifático calculados a partir da técnica integrável de ${ }^{13}$ C GATED inverso.

\begin{tabular}{ccc}
\hline Amostra & \% de carbono aromático & \% de carbono alifático \\
\hline CAP & 18,78 & 81,22 \\
Borra & 28,41 & 71,59 \\
\hline
\end{tabular}

Verificou-se um aumento na porcentagem de carbono aromático da borra em relação ao CAP.

\section{Infravermelho (FTIR)}

A caracterização estrutural foi analisada observando-se os espectros na região do infravermelho (FTIR) do CAP e da borra, como mostra a Figura 3.

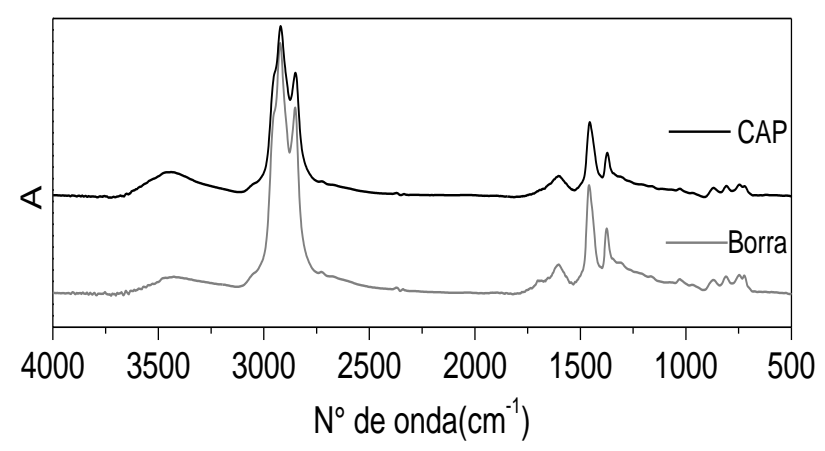

Figura 3- Espectro de FTIR em pastilha de KBr do CAP e da borra.

Observa-se, a partir da análise dos espectros, as seguintes bandas características para o CAP e a borra [26,27]: 2920 e $2848 \mathrm{~cm}^{-1}$, relativas à presença de estiramentos $\mathrm{C}-\mathrm{H}$; as bandas em $1635-1753 \mathrm{~cm}^{-1}$ relativas à formação de carbonila; em $1604 \mathrm{~cm}^{-1}$, relativas ao estiramento $\mathrm{C}=\mathrm{C}$ de estruturas aromáticas; em 1457 e $1379 \mathrm{~cm}^{-1}$, as de deformações $\mathrm{CH} 2$ e $\mathrm{CH} 3$ de estruturas alifáticas; $1031 \mathrm{~cm}^{-1}$, decorrente da presença de grupamentos sulfóxidos; 871 e $879 \mathrm{~cm}^{-1}$ caracterizados pelas deformações de $\mathrm{C}-\mathrm{H}$ de estruturas aromáticas substituídas. 
A borra apresentou, além das bandas características, um aumento da absorção na frequência de $1700 \mathrm{~cm}^{-1}$ e $1030 \mathrm{~cm}^{-1}$ atribuídas ao estiramento $C=O$ e ao estiramento $S=0$. Dessa forma, fica evidente uma pré-concentração de substâncias que possuam estes grupamentos, pois este tipo de resíduo apresenta na sua composição óleo, água e sólidos. Além disso, essa amostra sofre um aquecimento prolongado com liberação de voláteis, conferindo características bastante variáveis. Este mesmo comportamento foi verificado quando da análise dos HPAs.

\section{Análise Termogravimétrica}

A Figura 4 mostra as curvas de decomposição a $10^{\circ} \mathrm{C} / \mathrm{min}$ do CAP e da borra.

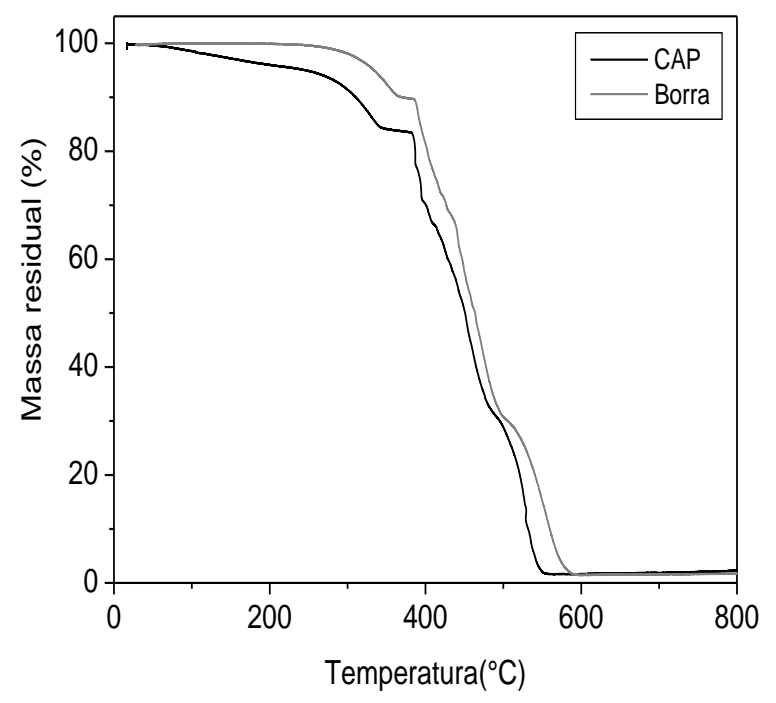

Figura 4- Curvas Termogravimétricas do CAP e da borra em atmosfera oxidativa.

O comportamento das amostras analisadas foi semelhante. Em atmosfera oxidativa todos apresentaram 4 eventos. Não foi observada diferença considerável no teor de resíduos e no número de eventos da decomposição térmica quando o CAP e a borra foram submetidos à atmosfera oxidativa. A borra possui, além dos hidrocarbonetos presentes no CAP, uma composição de silicatos (argila e lama), água produzida, óleo e produtos químicos utilizados no processo de extração [28] o que, de certa forma, conferiu uma maior estabilidade térmica quando comparada com o CAP.

\section{CONCLUSÕES}

As seguintes observações e conclusões podem ser citadas com relação ao comportamento obtido nos ensaios químicos realizados com CAP convencional e borra oleosa asfáltica pura:

a) Os espectros de FTIR indicaram a presença dos grupamentos aromáticos, alifáticos, carbonilas e sulfóxidos, entre outros, concordantes com a presença de compostos naftênicos, aromáticos, saturados e olefinas, característicos dos cimentos asfálticos e que também foram observados na borra. Pela análise dos espectros de RMN 1H, o CAP e a borra apresentam em suas estruturas grupamentos aromáticos, alifáticos e/ou cíclicos. 
b) A análise das curvas termogravimétricas do CAP e da borra mostrou comportamento semelhante. Os eventos, no entanto, ocorreram em temperaturas distintas.

c) Os asfaltenos são a fração mais pesada dos CAPs estudados, sendo que a borra apresentou um valor maior das frações de massa molar, quando comparada ao CAP.

d) Tanto o CAP quanto a borra oleosa apresentou HPAs considerados tóxicos e outros considerados mutagênicos e carcinogênicos, além de outros HPAs, diferentes dos padrões analíticos estudados. Os valores de concentração para HPAs considerados tóxicos, mutagênicos e carcinogênicos, são maiores para a borra oleosa, com exceção dos HPAs: m-naftaleno, fluoreno, fluoranteno, criseno, benzo(b)fluoranteno e benzo(k)fluoranteno.

\section{AGRADECIMENTOS}

Os autores querem expressar seus agradecimentos ao CNPq, à CAPES pelo apoio financeiro, bem como à Lubnor/Petrobras pelo fornecimento do CAP e disponibilização da borra asfáltica para o estudo realizado e ao CENAUREM pela realização das análises de RMN.

\section{REFERÊNCIAS BIBLIOGRÁFICAS}

1. ARAÚJO, L. S. A., NICOLAIEWSKY, E., FREIRE, D. D. C.O Estudo de Caso do Gerenciamento de Resíduos Sólidos em uma Refinaria de Petróleo. In: $2^{\circ}$ Congresso Brasileiro em Petróleo e Gás (P\&D), Rio de Janeiro-RJ, 2003.

2. GBADEGESIN, M. A., ODUNOLA, O. A., AKINWUMI, K. A., OSIFESO, O. O. Comparative hepatotoxicity and clastogenicity of sodium arsenite and three petroleum products in experimental Swiss Albino Mice: the modulatory effects of Aloe vera gel. Food and Chemical Toxicology, v. 47, n.10, p. 2454-2457, 2009.

3. CUNHA, C. E. S. C. P. Gestão de resíduos perigosos em refinarias de petróleo. Dissertação de Mestrado. Rio de Janeiro-RJ, 2009. Universidade do Estado do Rio de Janeiro, 2009.

4. MAGALHÃES, S. C. Plano de gerenciamento de resíduos sólidos para a indústria de petróleo: o caso de Petrobras/Lubnor-CE. Dissertação de Mestrado, Fortaleza-CE, 2006. Universidade Federal do Ceará, 2006.

5. SANTOS, R. S., SOUZA, G. P., HOLANDA, J. N. F. Caracterização de massas argilosas contendo resíduo proveniente do setor petrolífero e sua utilização em cerâmica estrutural. Cerâmica, v. 48, n. 307, p.115-120, 2002.

6. ABNT- ASSOCIAÇÃO BRASILEIRA DE NORMAS TÉCNICAS. Resíduos sólidos - classificação, NBR 10004, Rio de Janeiro, 2004.

7. KUANG, S., XU, Z. Pollution characteristics of polycyclic aromatic hydrocarbons (PAHs) in oily sludge from the Zhongyuan Oilfield and its peripheral soils. Chinese Journal of Geochemistry, v. 28, n. 2, p. 176-183,jun. 2009.

8. BINET, S., PFOHL-LESZKOWICZ. A., BRANDT. H., LAFONTAINE M., CASTEGNARO M. Bitumen fumes: review of work on the potential risk to workers and the present knowledge on its origin. Science of the Total Environment, v. 300, n. 1-3, p.37-49, 2002.

9. LOPES, J. L. Riscos para a saúde de trabalhadores de pavimentação com asfalto. Revista de Gestão Integrada em Saúde do Trabalho e Meio Ambiente, v.3, n.3, Seção Interfacehs1, ago./ dez. 2008. 
10. Institute of Petroleum. Standard Methods for Analysis and Testing of Petroleum and Related Products, IP 143, 1989.

11. KARACAN, O., KOK, M., KARAASLAN, U. U. Changes in thermal Properties of crude oil asphaltenes induced by $\mathrm{NaOH}$ treatment. Fuel Processing Technology, v.59, n. 1, p. 13-21, 1999.

12. FERNANDES, P. R. N. Caracterização de Ligante Asfáltico Brasileiro e Avaliação da Presença de Hidrocarbonetos Políciclicos Aromáticos (HPAs). Dissertação de Mestrado. Fortaleza-CE, 2007. Universidade Federal do Ceará, 2007.

13. PINHEIRO, L. S., FERNANDES, P. R. N., CAVALCANTE, R. M., NASCIMENTO, R. F., SOARES, J. B., SOARES, S. A., FREIRE, J. A. K. Polycyclic aromatic hydrocarbons from asphalt binder: Extraction and characterization. Journal of the Brazilian Chemical Society, v.20, n.2, p.222228, 2009.

14. SILVA, A. A. R., LOBO, I., GUEDES, C. L. B., PINTO, J. P. Análise de Hidrocarbonetos Policíclicos Aromáticos (HPAs) em solos utilizando agitação ultra-sônica, tubo aquecedor/minicondensador e cromatografia gasosa. Semina: Ciências Exatas e Tecnológicas, v.27, n.2, p.105-111, jul./dez. 2006.

15. LANÇAS, F. M. The Role of the Separation Sciences in the $21^{\text {th }}$ Century. Journal of the Brazilian Chemical Society, v. 14, n. 2, p. 183-197.

16. CAVALCANTE, R. M. Distribuição, fluxo deposicional e estimativa de fontes de HPAs na cidade de Fortaleza. Tese de Doutorado. Fortaleza-CE, 2007. Universidade Federal do Ceará, 2007.

17. FERNANDES, P. R. N., SOARES, S. A., NASCIMENTO, R. F., SOARES, J. B., CAVALCANTE, R. M. J. Evaluation of polycyclic aromatic hydrocarbons in asphalt binder using matrix solid-phase dispersion and gas chromatography. Journal of Chromatographic Science, v.47, n. 9, p. 789793, 2009.

18. IARC- International Agency for Research on Cancer. Some traditional herbal medicines, naphthalene and styrene. In: Monographs on the Evaluation of Carcinogenic Risks to Humans, vol. 82. France: International Agency for Research on Cancer Published, 2002, 590p.

19. EPA - U. S. Environmental Protection Agency. IntegratedRisk Information System. IRIS Substance List. Disponível em: www.epa.gov/iris/subst/index.html. Acesso em: 25 de janeiro de 2013.

20. LIMA, A. da C. Determinação de hidrocarbonetos policíclicos aromáticos em águas superficiais nos Rios Piracicaba e Doce, Ipatinga-MG, utilizando a técnica de EFS-CLAE-DAD. Dissertação de Mestrado. Ouro preto-MG, 2008. Universidade Federal de Ouro Preto, 2008.

21. Brasil. Ministério da Saúde. Portaria Ministerial № 518 de 25 de março de 2004. LEX: DOU 26/03/2004, Seção 1, pág. 266. Disponível em WWW.água.org.br. Acesso em: em 25 de janeiro de 2013.

22. SHRP- Strategic Highway Research Program A-335. Superior Performing Asphalt Pavement. Superpave: The Product of SHRP Asphalt Research Program. Binder Characterization and Evaluation by Nuclear Magnetic Resonance Spectroscopy, National Research Council, Washington, DC, 1993. 
23. BORREGO, A.G., BLANCO, C. G., PRADO, J. G., DÍAZ, C., GUILLÉN, M. D. ${ }^{1}$ H NMR and FTIR Spectroscopic studies of bitumen and shale oil from selected Spanish oil shales. Energy \& Fuels, v.10, n.1, p. 77-84, 1996.

24. SHAKIRULLAH, M., AHMAD, I., RAHMAN, H., KHAN, M. A., ISHAQ, M., SHAH, A. A. Spectral Analysis of some distillates from asphalt cracking and evaluation of UTIMAC as cracking catalyst. Journal of the Chinese Chemical Society, v. 53, p. 1059-1066, 2006.

25. MICHON, L., MARTIN, D., PLANCHE, J. P. E. Estimation of average structural parameters of bitumens by $13 \mathrm{C}$ nuclear magnetic resonance spectroscopy. Fuel, v. 76, n. 1, p. 9-15, 1997.

26. MASSON, J. F., PELLETIER, L., COLLINS, P. Rapid FTIR method for quantification of styrenebutadiene type copolymers in bitumen. Journal of Applied Polymer Science, v. 79, n. 6, p. 1034-1041, 2001.

27. LAMONTAGNE, J., DUMAS, P., MOUILLET, V., KISTER, J. Comparison by Fourier transform infrared (FTIR) spectroscopy of different ageing techniques: application to road bitumens. Fuel, v. 80, n. 4, p. 483-488, 2001.

28. ALVES, M. R. F. V. Reciclagem de borra oleosa: Uma contribuição para a gestão sustentável dos resíduos da indústria de petróleo em Sergipe. Dissertação de Mestrado. São Cristovão-SE, 2003. Universidade Federal de Sergipe, 2003. 